\title{
Persistence of Neutralizing Antibody Against Dengue Virus 2 After 70 Years from Infection in Nagasaki
}

\author{
Mya Myat Ngwe Tun,"* Yoshihito Muta?, Shingo Inoue, and Kouichi Morita ${ }^{1}$
}

\begin{abstract}
This study aimed to investigate the duration of humoral immune responses to dengue virus (DENV) infection in Japanese who experienced acute febrile illness with hemorrhagic manifestations 70 years ago, when an epidemic of dengue occurred in Nagasaki, Japan, from 1942 to 1944. A Japanese volunteer requested serological diagnosis of DENV infection in 2014 and donated blood sample to measure the antibody titer against DENV by antiflavi IgG indirect ELISA, focus reduction neutralization test, and plaque reduction neutralization test. The serum sample of the volunteer was positive in flavi IgG ELISA and it indicated primary infection. In the neutralization test, the highest neutralizing titer was $\geq 218$ for DENV-2. We report here the existence of DENV-specific antibodies in the serum of a person after 70 years from infection. Published reports indicated that DENV-1 was responsible for the 1942-1944 outbreak in Nagasaki. However, our data suggested that DENV-2 also played a role in this Nagasaki dengue epidemic.
\end{abstract}

Key words: dengue; Japan; neutralization antibody

\section{Introduction}

Dengue virus (DENV) is a member of the genus Flavivirus, family Flaviviridae. DENV is transmitted by infected mosquitoes, mainly Aedes aegypti and Aedes albopictus. The four serotypes of DENV (DENV-1 to DENV-4) are antigenically and genetically distinct. Infection by any DENV serotype can cause mild dengue fever (DF), severe dengue hemorrhagic fever, or the more severe dengue shock syndrome, as described in the 1997 World Health Organization classification system of the disease severity. This is now categorized into dengue with or without warning signs and severe dengue. $^{1}$

DENV infection is found in tropical and subtropical regions around the world such as Southeast and South Asia, Pacific, Central and South America, the Caribbean, Africa, and Europe. In Japan, dengue epidemics were recorded in the Main Islands (1942-1945) once before the end of World War II and several times (1893-1955) in the Okinawa Islands. ${ }^{2,3}$ A dengue outbreak due to DENV-1 occurred in Osaka, Kobe, Hiroshima, and Nagasaki from 1942 to $1945 .{ }^{4}$ In Okinawa, the outbreak was due to DENV-1 and DENV-2. After several decades without confirmed autochthonous cases of DF in Japan, a patient with no history of overseas travel was reported to contract DF in Tokyo, and as of October 2014, a total of 160 autochthonous cases were confirmed in this country. ${ }^{6}$ This study aimed to investigate the duration of humoral immune responses to DENV infection in a Japanese person who experienced acute febrile illness with hemorrhagic manifestations 70 years ago, when an epidemic of dengue occurred in Nagasaki, Japan, from 1942 to 1944.

\footnotetext{
${ }^{1}$ Department of Virology, Institute of Tropical Medicine, Leading Graduate School Program, Nagasaki University, Nagasaki, Japan.

${ }^{2}$ Muta Hospital, Omura City, Japan.

*Address correspondence to: Mya Myat Ngwe Tun, PhD, Department of Virology, Institute of Tropical Medicine, Nagasaki University, 1-12-4 Sakamoto, Nagasaki City 8528523, Japan, E-mail: myamyat@tm.nagasaki-u.ac.jp
}

(c) Mya Myat Ngwe Tun et al. 2016; Published by Mary Ann Liebert, Inc. This Open Access article is distributed under the terms of the Creative Commons License (http://creativecommons.org/licenses/by/4.0), which permits unrestricted use, distribution, and reproduction in any medium, provided the original work is properly credited. 


\section{Materials and Methods}

Viruses and cell lines

The viruses used for anti-flavi IgG ELISA, focus reduction neutralization test $\left(\mathrm{FRNT}_{50}\right)$, and plaque reduction neutralization test $\left(\mathrm{PRNT}_{50}\right)$ were as follows: DENV-1 (strain: M-120), DENV-2 (strain: M-58), DENV-3 (strain: SLMC50), DENV-4 (strain: SLMC318), and Japanese encephalitis virus (strain: JaOArS982). These viruses were propagated in the $\mathrm{C} 6 / 36$ mosquito cell line and were used to inoculate the Vero and baby hamster kidney (BHK) cell lines for virus titration and neutralization tests.

\section{Detection of flavi lgG}

In-house flavi IgG indirect ELISA was carried out following the protocol described previously. ${ }^{7,8}$ The plate was coated with purified Japanese encephalitis virus (JEV) antigen ( $250 \mathrm{ng} / 100 \mu \mathrm{L} /$ well $)$ and incubated at $4^{\circ} \mathrm{C}$ overnight. Wells were blocked with Block Ace and were incubated at room temperature (RT) for $1 \mathrm{~h}$. After incubation, wells were washed with phosphatebuffered saline (PBS) containing 0.05\% Tween 20 (PBS$\mathrm{T})$ three times. Test samples and positive and negative controls were diluted 1:1000 in PBS-T and $100 \mu \mathrm{L}$ aliquots were distributed into duplicate wells. The plate was incubated at $37^{\circ} \mathrm{C}$ for $1 \mathrm{~h}$ and then washed as already described. After washing, 1:30,000 dilution of HRP-conjugated antihuman IgG (American Qualex) was added at $100 \mu \mathrm{L} /$ well and plate incubated at $37^{\circ} \mathrm{C}$ for $1 \mathrm{~h}$. The plate was washed and $o$-phenylenediamine dihydrochloride substrate at $100 \mu \mathrm{L} /$ well was added and kept in the dark at RT for $1 \mathrm{~h}$. To terminate the reaction, $100 \mu \mathrm{L}$ of $1 \mathrm{~N}$ sulfuric acid was added to each well.

The optical density (OD) was read at $492 \mathrm{~nm}$ (Multiscan JX). A standard curve was prepared using the OD values of the dengue-positive control serum samples starting with a 1000 -fold dilution, followed by serial 2-fold dilutions. A sample titer equal to or greater than 1:3000 was considered to be positive. DENV primary and secondary infections were determined at a sample titer $<52,000$ and $\geq 52,000$, respectively.

\section{Focus reduction neutralization test}

To confirm neutralizing activity of DENV and JEVspecific antibodies, neutralization tests were done. ${ }^{9}$ Patient serum samples were heat inactivated at $56^{\circ} \mathrm{C}$ for $30 \mathrm{~min}$ before the assay. Serum samples were serially diluted twofold with $2 \%$ fetal calf serum (FCS) in Eagle's minimum essential medium (MEM). A volume of $150 \mu \mathrm{L}$ of each dilution of the serum samples was mixed with an equal volume of DENV or JEV, which contained 60 focus-forming units. This was followed by incubation at $37^{\circ} \mathrm{C}$ for $1 \mathrm{~h}$ for a virus-antibody neutralization reaction. The virus and serum mixture was inoculated onto Vero cell monolayer in a 96 -well plate at $37^{\circ} \mathrm{C}$ for $1 \mathrm{~h}$. After incubation, the infected cells were overlaid with $1.25 \%$ methylcellulose 4000 in 2\% FCS in MEM. The plates with JEV and with DENV were then incubated at $37^{\circ} \mathrm{C}$ for $36 \mathrm{~h}$ and 3 days, respectively. The plates were washed with PBS, fixed with $4 \%$ paraformaldehyde phosphate buffer solution for $30 \mathrm{~min}$ at RT, rinsed, and cells in each well were permeabilized with $1 \%$ NP-40 solution in PBS for $30 \mathrm{~min}$ at RT. After washing, the plates were blocked with Block Ace for $30 \mathrm{~min}$ at RT. A pool of human serum samples with a high titer for antiflavivirus IgG (diluted 1:1500) was then added, and plates were incubated at $37^{\circ} \mathrm{C}$ for $1 \mathrm{~h}$ and washed. Subsequently, 1:1500-diluted HRP-conjugated goat antihuman IgG was added to the plates and incubated at $37^{\circ} \mathrm{C}$ for $1 \mathrm{~h}$. Staining of cells was done by the addition of a $0.5 \mathrm{mg} /$ $\mathrm{mL}$ solution of substrate $3,3^{\prime}$-diaminobenzidine tetrahydrochloride in PBS with $0.03 \%$ of $\mathrm{H}_{2} \mathrm{O}_{2}$, and the staining reaction was allowed to proceed for $10 \mathrm{~min}$ at RT. After washing the stained cells, the number of foci of stained cells per well was counted under a microscope.

The reciprocal of the endpoint serum dilution that provided a $50 \%$ or greater reduction in the mean number of foci relative to the control wells that contained no serum was considered to be the $\mathrm{FRNT}_{50}$ titer. On the basis of the previously published criteria of neutralizing antibody patterns, ${ }^{8,10}$ a monotypic antibody response (primary infection) was defined as neutralization titer $\geq 1: 10$ to only one serotype, or neutralization titer $\geq 1: 10$ for more than one serotype but 1:80 for only one serotype. In monotypic pattern of antiDENV neutralizing antibodies, the DENV serotype with the highest $\mathrm{FRNT}_{50}$ was assumed to be the infecting serotype. Multitypic antibody response (secondary infection) was defined as neutralization titer $>10$ for more than one serotype without a neutralization titer $\geq 80$ to only one serotype.

\section{Plaque reduction neutralization test}

Heat-inactivated human serum samples were serially diluted twofold in 2\% FCS in MEM. One hundred fifty microliters of each dilution of the serum samples was mixed with an equal volume of virus, which contained 60 plaque-forming units, followed by incubation at $37^{\circ} \mathrm{C}$ for $1 \mathrm{~h}$ for a virus-antibody neutralization reaction. One hundred microliters of each mixture 
Table 1. Neutralization Antibodies Profile of a Japanese Person Who Reported Experiencing Dengue Fever-Like Illness in 1944

\begin{tabular}{cccccccrrr}
\hline Patient & Age (year) & Sex & Flavi lgG titer & $\begin{array}{c}\text { Neutralization } \\
\text { test }\end{array}$ & $\begin{array}{c}\text { DENV-1 } \\
\text { M-120 }\end{array}$ & $\begin{array}{c}\text { DENV-2 } \\
\text { M-58 }\end{array}$ & $\begin{array}{c}\text { DENV-3 } \\
\text { SLMC50 }\end{array}$ & $\begin{array}{c}\text { DENV-4 } \\
\text { SLMC318 }\end{array}$ & $\begin{array}{c}\text { JEV } \\
\text { S982 }\end{array}$ \\
\hline 1 & 83 & M & 31,083 & FRNT $_{50}$ & 71 & 218 & 30 & 32 \\
\end{tabular}

DENV-1 M-120, Myanmar strain (M-120/2013); DENV-2 M-58, Myanmar strain (M-58/2013); DENV-3 SLMC 50, Philippine strain (SLMC50); DENV-4 SLMC 318, Philippine strain (SLMC318); FRNT $50,50 \%$ focus reduction neutralization test; JEV-S982, Japan strain (JaOArS982); PRNT $50,50 \%$ plaque reduction neutralization test.

was then used to inoculate BHK cell monolayer in a 24well plate. After incubation at $37^{\circ} \mathrm{C}$ for $1 \mathrm{~h}$, the infected cells were overlaid with $500 \mu \mathrm{L}$ of MEM containing $2 \%$ FCS and $1 \%$ methylcellulose. The plates were then incubated at $37^{\circ} \mathrm{C}$ for $3-5$ days based on the virus and dengue serotypes. The plate was washed with PBS to remove methylcellulose, fixed with $4 \%$ paraformaldehyde phosphate buffer solution, and stained with $0.1 \%$ crystal violet in $10 \%$ ethanol. The reciprocal of the endpoint serum dilution that provided a $50 \%$ or greater reduction in the mean number of plaque relative to the control wells that contained no serum was considered to be the $\mathrm{PRNT}_{50}$ titer.

\section{Results and Discussion}

We investigated the duration of humoral responses to DENV infection of an 84-year-old Japanese male, who experienced acute febrile infection with hemorrhagic manifestations when he was a 13-year-old junior high school student in Nagasaki in August 1944. During that period, there was a dengue outbreak in Nagasaki. He was clinically diagnosed to have DF. His classmates and his neighbors experienced clinical symptoms similar to what he had, such as severe headache, fatigue, muscle pain, high fever, severe nasal bleeding, vomiting with blood, and bloody stool. After 70 years from this experience, this person requested serological diagnosis of DENV infection in October 2014 and volunteered to donate his blood sample. The volunteer has no travel history to dengue endemic countries and he resided in a place where there were no autochthonous cases of dengue. He had also not experienced the same symptoms of dengue for the second time. He has a JEV vaccination history.

Table 1 shows the results of ELISA, $\mathrm{FRNT}_{50}$, and $\mathrm{PRNT}_{50}$ of the serum sample. The serum sample of the volunteer was flavi IgG ELISA positive, and based on the IgG titer, it indicated primary infection. In the neutralization test, the highest neutralizing titer was found in DENV-2. Presence of DENV-1, -3, and -4, and JEV neutralizing antibodies was detected, but their levels were low. Based on the previously published criteria of monotypic pattern of anti-DENV neutralizing antibodies, the DENV serotype with the highest neutralization titer was assumed to be the infecting serotype. Thus, it is most likely that the patient was infected with DENV-2.

In several studies, long-term existence of antiflavivirus antibodies has been reported. Fujita et al. ${ }^{11}$ mentioned that in their study one of the patients who stayed in Nagasaki had DENV-2 neutralizing titer a little higher than DENV-1. The question of whether the DENV-2 was involved in the Nagasaki epidemic deserves further consideration. ${ }^{11}$ Taken together, our data strongly suggest that DENV-2 played a role in the Nagasaki dengue epidemic in 1942-1944. The long-term persistence of antiflavivirus neutralizing antibody up to 30-60 years has been demonstrated in several studies. ${ }^{12}$ We report here the existence of antibody against DENV in the serum of a person who got infected 70 years ago.

\section{Acknowledgments}

We thank the valuable scientific suggestions of Corazon Cerilla Buerano and the support of the members of the Department of Virology, Institute of Tropical Medicine, Nagasaki University. This work was supported by the government of Japan through the grant-in-aid for Scientific Research (KANKENHI) and partially supported by Japan Initiative for Global Research Network on Infectious Diseases from Japan Agency for Medical Research and Development (AMED); Science and Technology Research Partnership for Sustainable Development (SATREPS) Project. This work was partly conducted at the Joint Usage Research Centre on Tropical Diseases, Institute of Tropical Medicine, Nagasaki University.

\section{Author Disclosure Statement}

No competing financial interests exist. 


\section{References}

1. World Health Organization (WHO). Dengue Guidelines for Diagnosis, Treatment, Prevention, and Control. Geneva, 2009.

2. Schlesinger RW. Dengue Viruses. Virology Monographs. Springer-Verlag: New York, NY; pp. 8-13; 1977.

3. Hotta S. Experimental studies on dengue. I. Isolation, identification and modification of the virus. J infect Dis. 1952;90:1-9.

4. Hotta S. Twenty years of laboratory experience with dengue virus. In: Medical and Applied Virology. Saunders M, Lennette EH, (eds.) Green: St Louis; 1965.

5. Tadano M, Okuno Y, Fukunaga T, et al. Retrospective serological studies on dengue epidemics in Osaka and Okinawa. Biken J. 1983;26:165-167.

6. Kutsuna S, Kato Y, Moi ML, et al. Autochthonous dengue fever, Tokyo, Japan, 2014. Emerg Infect Dis. 2015;21:517-520.

7. Inoue $S$, Alonzo MT, Kurosawa $Y$, et al. Evaluation of a dengue IgG indirect enzyme-linked immunosorbent assay and a Japanese encephalitis IgG indirect enzyme-linked immunosorbent assay for diagnosis of secondary dengue virus infection. Vector Borne Zoonotic Dis. 2010;10: 143-150.

8. Ngwe Tun MM, Thant KZ, Inoue S, et al. Serological characterization of dengue virus infections observed among dengue hemorrhagic fever/ dengue shock syndrome cases in upper Myanmar. J Med Virol. 2013;85:1258-1266.

9. Ngwe Tun MM, Inoue $S$, Thant $K Z$, et al. Retrospective seroepidemiological study of chikungunya infection in South Asia, Southeast Asia and the Pacific region. Epidemiol Infect. 2016;144:2268-2275.

10. Endy TP, Nisalak A, Chunsuttitwat $S$, et al. Relationship of preexisting dengue virus (DV) neutralizing antibody levels to viremia and severity of disease in a prospective cohort study of DV infection in Thailand. J Infect Dis. 2004;189:990-1000.
11. Fujita N, Yoshida K. Follow-up studies on dengue endemic in Nagasaki, Japan: Detection of specific antibodies in the serum taken more than 30 years after a single attack of dengue. Kobe J Med Sci. 1979;25: 217-224.

12. Imrie A, Meeks J, Gurary A, et al. Antibody to dengue 1 detected more than 60 years after infection. Viral Immunol. 2007;20:672-675.

Cite this article as: Ngwe Tun MM, Muta Y, Inoue S, Morita K (2016) Persistence of neutralizing antibody against dengue virus 2 after 70 years from infection in Nagasaki, BioResearch Open Access 5:1, 188-191, DOI: 10.1089/biores.2016.0016.

\section{Publish in BioResearch Open Access}

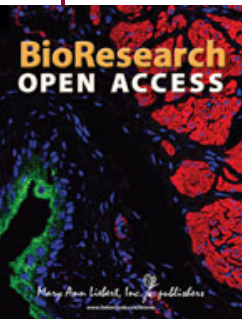

- Broad coverage of biomedical research - Immediate, unrestricted online access - Rigorous peer review - Compliance with open access mandates - Authors retain copyright

- Highly indexed

- Targeted email marketing

liebertpub.com/biores 